\title{
Memoria y adquisición del conocimiento
}

\section{Juan José Aparicio y José Luis Zaccagnini}

Universidad Complutense de Madrid

\section{$L$}

La Psicología se preocupó en sus comienzos por problemas importantes, tales como la adquisición, almacenamiento y utilización del conocimiento. En aquella época, sin embargo, nadie encontró el modo de abordar este tipo de cuestiones con un cierto rigor y los psicólogos cayeron en la trampa de desviar su atención hacia temas que pudieran tratarse con objetividad científica aun cuando carecieran de interés (1). Así, Ebbinghaus llevó a cabo una serie de trabajos sobre el aprendizaje de sílabas sin sentido, que quizá fuera la única forma de adquisición del conocimiento que pudiera estudiarse de un modo experimentalmente ortodoxo en aquel momento, pero que desde luego resultó ser posible. mente el tipo de investigación más desafortunada para conseguir averiguar cómo adquieren de hecho las personas el conocimiento. En palabras de Jenkins (1974) «Ebbinghaus cometió un error especialmente funesto. Su método, sus materiales y su orientación se combinaron para ocultar los de otra manera abrumadores efectos de la variable más poderosa en el funcionamiento natural de la memoria: los efectos del conocimientò (pág. 2).

Ebbinghaus no se planteó a fondo el problema de la adquisición y almacena- miento del conocimiento, porque consiguió eludir en gran medida con sus sílabas sin sentido algo que parecía ineludible, la utilización que las personas hacen del conocimiento que poseen del mundo cuando procesan la información. No obstante, sus trabajos iniciaron una tradición en el terreno del aprendizaje humano y la memoria que se prolongó durante muchos años. Con dicha tradición confluyeron algunas corrientes conductistas que dominaban el panorama en aquel tiempo. Como ejemplo de esta confluencia piénsese en el paradigma de pares asociados, según el cual el par de izquierda era un estímulo y el de la derecha una respuesta. Hoy sabemos que cuando alguien aprende un par de sílabas o un par de palabras no está aprendiendo una respuesta - el par de la derecha- a un estímulo - el par de la izquierda- (Greeno, 1970), pero este punto de vista sirvió durante algunas décadas para interpretar los fenómenos de aprendizaje verbal humano en términos de relaciones entre estímulos y respuestas.

Aunque hay antecederites notables, como el famoso artículo de Miller (1956) o el libro de Broadbent (1958), es sólo a partir de mediados de los años 60 cuando los estudios sobre la memoria humana asi- 
milaron de un modo ya más definitivo el sistema conceptual procedente de la ciencia de las computadoras. Esta estructura conceptual se ha mostrado más útil para describir los fenómenos de memoria y aprendizaje humano que los conceptos que tradicionalmente habían servido de soporte a los trabajos sobre aprendizaje animal (2). Todavía, sin embargo, existía un cierto hiato entre los estudios de memoria propiamente dichos y los estudios acerca de la adquisición del conocimiento. En los años 70 se ha roto esta barrera, de forma que estos dos grandes temas de la Psicología se presentan en este momento íntimamente relacionados. Con este artículo y con los dos que vienen a continuación pretende describirse este estado de cosas.

Para lograr este propóstio podría empezarse por una pregunta muy simple: ¿Qué debe hacer una persona si quiere aprenderse algo, por ejemplo, un teléfono? La mayor parte de la gente respondería que lo que tiene que hacer es repetir muchas veces el número de teléfono hasta que se lo aprenda. Los profesionales de la enseñanza durante muchos años también han empleado métodos derivados de este punto de vista. En realidad, no sólo el hom. bre de la calle o los profesores, sino también la mayoría de los psicólogos llegaron a pensar que la simple repetición de un item bastaba para que éste pudiera ser retenido. Una de las teorías más comúnmente aceptadas derivaba del modelo de aprendizaje animal de Hull-Spence, según el cual cada vez que se volvía a presentar un item a un sujeto se producía un incremento en la fuerza de la huella de memoria. Así, Underwood y Keppel (1962) pensaban que la fuerza de la huella iba aumentando de un modo continuo con cada repetición.

La primera reacción contra esta simplificación partió de autores como Estes (1960) o Bower (1962) que, aun manteniendo la hipótesis de que la cantidad de repeticiones era decisiva para el aprendizaje, no pensaban, sin embargo, que éste se pro- dujera de un modo continuo, sino de un modo discontinuo. Según estos teóricos, un item nó se aprendía progresivamente, sino que se aprendía o no se aprendía. El número de presentaciones de un item era importante, porque al repetirse muchas veces se aumentaba la probabilidad de que dicho item pasara de un estado de no aprendido a un estado de aprendido. Como el lector habrá reconocido, estas teorías del aprendizaje son las que se conocen con el nombre del todo o nada. Lo que aquí interesa señalar en relación con ellas es que la memorabilidad de un item ya no dependía únicamente de factores cuantitativos, sino que éstos -el número de repeticiones- aumentaban la probabilidad de que el item adquiriese un estado cualitativamente distinto.

Los ya clásicos trabajos de Brown (1958) en Inglaterra y Peterson y Peterson (1959) en América sobre la influencia de tareas interpoladas entre la presentación de un item y su recuperación en un espacio corto de tiempo, junto con los de Murdock (1962) sobre la curva de posición serial consolidaron la noción de memoria a corto plazo. Incoporando esta noción, Atkinson y Crothers (1964) añadieron a los dos estados de no aprendido y aprendido un estado intermedio que se corresponde con la memoria a corto plazo. A partir de ahí las explicaciones sobre los fenómenos de memoria iban a tomar un sesgo claramente estructural. Waugh y Norman (1965) y Atkinson y Shiffrin (1968) fueron más allá en la idea de los estados de memorabilidad postulando distintos tipos de almacenes en los que se localiza la información, de manera que la memorabilidad de un item depende del almacén en que se encuentre. En esencia, al margen del almacén de memoria sensorial (3), se proponía por un lado la existencia de un almacén a corto plazo de capacidad limitada, en donde la información se representaba auditivamente y donde se perdía al ser desplazada por otra información que entraba. Por otro, un almacén a largo plazo, que 
tenía teóricamente una capacidad ilimita$\mathrm{da}$, en donde la información se representaba de un modo significativo, abstracto y organizado, y se perdía por interferencia o simplemente dejaba de ser recuperable por alguna razón que imposibilitara su acceso. Simplificando un poco las cosas, la memoria a largo plazo sería el conocimiento estructurado del mundo que posee un individuo. Pero volvamos al almacén a corto plazo: un modo de mantener la información en dicho almacén consistía en entregarse a actividades de repaso (rehearsal). Si una información dejaba de repasarse era desplazada de la memoria a corto plazo por otra información y se perdía, a no ser, claro está, que hubiese pasado al almacén a largo plazo.

Retomando nuestro ejemplo, cuando una persona intenta aprenderse un teléfono, lo que tendría que hacer, según lo dicho hasta ahora, es mantenerlo en el almacén a corto plazo y, para ello, repasarlo, es decir, repetírselo a sí mismo, hasta que entrara en la memoria a largo plazo. El teléfono queda, pues, aprendido cuando está en la memoria a largo plazo y el sujeto no tiene que hacer nada para mantenerlo. Por lo tanto, aprender algo es en último término incorporar ese algo a la memoria a largo plazo (4); de ahí que explicar cómo tiene lugai la adquisición del conocimiento sea fundamentalmente explicar cómo pasa a formar parte de la memoria a largo plazo la información proveniente del medio. Desde este punto de vista, la transferencia de la información de la memoria a corto plazo a la memoria a largo plazo se convierte en un problema crucial. Habría que preguntarse entonces cuál era el factor más importante que determinaba el paso de la información de un almacén a otro para los teóricos de los años 60. La respuesta no podía ser más desalentadofa; con mayor o menor complejidad, el factor decisivo era la cantidad de tiempo que permanecía la información en la memoria a corto plazo o, en todo caso, la cantidad de tiempo que el sujeto empleaba para repasarla (5).

Naturalmente, lo que hay que saber es qué es lo que ocurre con la información durante el tiempo que permanece en la memoria a corto plazo para entender cómo pasa a la memoria a largo plazo, pero en aquellos años había una obsesión prematura por la matematización de los procesos mentales y es más fácil desarrollar un modelo matemático-formal cuando se manejan variables como el tiempo que cuando las yariables son los cambios cualitati. vos que sufre la información en el curso del procesamiento. Con la Psicología matemática ha sucedido algo parecido a lo que comentábamos de Ebbinghaus; como no se podían tratar matemáticamente las cuestiones importantes se acababan por expresar los aspectos más triviales con las más complicadas fórmulas matemáticas. Lo realizado en estos años, sin embargo, sentó las bases de desarrollos ulteriores. No hay que perder de vista que desde finales de los año 50 hasta finales de los 60 el interés se centró más en la memoria a corto plazo que en la memoria a largo plazo. Gracias a este interés se estudiaron algunos procesos que hoy se consideran básicos. Aparte del mecanismo de la atención, que en una de sus facetas selecciona la información que, procedente de la memoria sensorial, va a procesarse en la memoria a corto plazo, se estudiaron otros procesos de control como los ya mencionados de repaso y los de codificación. En cuanto a estos últimos tienen una doble vertiente. Por un lado hay que saber cómo se codifica una información para que entre a formar parte de la memoria a largo plazo, que es el tema de estudio al que nos dirigimos. Por otro, cómo debe codificarse una lista de items para aumentar al máximo la amplitud de la memoria a corto plazo. En relación con este segundo punto, desde el arrículo de Miller (1956) ya mencionado, sabemos que la estrategia consiste en conseguir agrupar la información en aproximadamente 7 porciones 
(chunks). En el caso de la memoria de dígitos, por ejemplo, somos capaces de repasar mentalmente 7 dígitos, a no ser que logremos hacer de los números de dos dígitos unidades significativas, en cuyo caso podríamos repasar mentalmente 14 dí. gitos. Asi, si queremos aprendernos dos números de teléfono al mismo tiempo tendremos que repasarlos agrupando los dígitos de dos en dos. A modo de inciso, y para completar el panorama, a finales de los años 40 y principios de los 50 irrum. pió de un modo un tanto efímero en la Psicología la Teoría de la Información, que pretendía establecer los límites en la capacidad de procesamiento en términos de bitios. Al introducir Miller la porción (chunk) como unidad de información en lugar del bitio no se limitó a sustituir una unidad por otra, sino que acabó con el intento de medir la capacidad de procesamiento humana en términos de la Teoría de la Información. La cantidad de información que puede procesar una persona no puede medirse del mismo modo que si se tratara de un ingenio mecánico por la sencilla razón de que los organismos no son "cajas negras», sino que son capaces de conferir un significado a la información.

Volviendo al problema del paso de la información a la memoria a largo plazo, el cambio que se ha producido en los años 70 tuvo lugar cuando se cayó en la cuenta de que entender la memoria a corto plazo o memoria primaria como un almacén no resultaba muy útil para explicar los datos de que se disponía. Lo que actualmente se intenta establecer no es la localización.de la información en una estructura para poder así completar un diagrama de flujo, sino la naturaleza de los procesos que hacen posible la incorporación de la información a la memoria a largo plazo. Así, el interés por la estructura global de la memoria ha decaído para dar paso al estudio acerca de los procesos. La pregunta no es ya cuáles son las etapas por las que atraviesa la información, sino qué operaciones realiza un individuo con la información.
Este cambio de perspectiva se inicia con el artículo de Craik y Lockhart que viene a continuación. Las objeciones que en él se dirigen a las teorías multialmacén (6) no deben interpretarse como una mera crítica para mejorar un modelo existente, sino que representa un cambio más radical con respecto al punto de vista anterior acerca del aprendizaje humano y la memoria. Por primera vez, el énfasis se pone en los procesos en detrimento de las descripciones estructurales. El tiempo de repaso o de permanencia en la memoria a corto plazo deja de ser un factor principal. Una información que se ha repasado durante largo tiempo puede no ser recuperada posteriormente, mientras que otra, que apenas ha sido repasada, se recupera con facilidad. La clave está en el nivel de profundidad a que se procese la información. Desde el punto de vista de los procesos de repaso lo que sucede es que puede repasarse una información simplemente para mantenerla o puede elaborarse para procesarla a un mayor nivel de profundidad, con lo que se facilitará su transferencia a la memoria a largo plazo. Esta es la distinción entre repaso del Tipo I, o de man. tenimiento, y repaso del Tipo II, o de elaboración, que introducen Craik y Lockhart.

Volvamos de nuevo al ejemplo del teléfono. Una peŕsona puede estar repitiéndose a sí misma un teléfono y no aprendérselo. Según la teoría de los niveles de Craik y Lockhart, tendrá que elaborar de alguna manera ese conjunto de números, en principio sin sentido, para poder asegurarse de que va a recordarlo en lo sucesivo sin tener que repasarlo constantemente. Es como cuando de jóvenes leíamos nuestras lecciones una y otra vez, y en unas ocasiones nos las aprendíamos y en otras no. Es decir, unas veces con las sucesivas lecturas conseguíamos elaborar la información procesándola a un mayor nivel de profundidad y otras veces nos limitábamos a mantenerla mientras duraba la lectura. A parte de que quizá no poseyé. ramos aún las estrategias adecuadas para 
memorizar aquellos textos, pasaba que en la inmensa mayoría de los casos lo que nos hacían aprender carecía en absoluto de sentido para nosotros y, por esta razón, nos resultaba difícil elaborar la información en profundidad por más que la releíamos. Porque procesar un material en profundidad quiere decir relacionarlo con nuestro conocimiento del mundo, y las cosas que una persona conoce dependen del sentido que esas cosas tengan para su vida. Más adelante se insistirá en esta cuestión.

Ahora es importante hacer notar que la memoria a largo plazo, es decir, el conjunto de experiencias que almacenamos a lo largo de nuestra vida, desempeña un papel principal en la adquisición de nuevos conocimientos, dado que desde el punto de vista funcional, la memoria a largo plazo es también el conjunto de esquemas de conocimiento de los que nos valemos para interpretar la realidad. El nuevo material se incorpora, pues, en función de lo que ya tenemos en nuestra mente. $\mathrm{O}$, para decirlo de otra manera, integramos las nuevas experiencias en relación con las experiencias pasadas. Ahora bien, si la memoria a corto plazo deja de ser un almacén para pasar a ser el conjunto de actividades que se llevan a cabo con el fin de incorporar la información a la memoria a largo plazo y, al mismo tiempo, las nuevas experiencias se asimilan en función de las experiencias pasadas, los contenidos de la memoria a corto plazo no serán otra cosa que el efecto de la activición temporal de algunos fragmentos de la memoria a largo plazo (Bjork, 1975; Shiffrin, 1975; Craik y Jacoby, 1975). La memoria a corto plazo, pues, no es una estructura con características fijas. Su naturaleza viene definida por el tipo de material que esté procesándose y por el tipo de procesamiento que está llevándose a cabo.

Como fácilmente puede adivinarse, el punto esencial de la teoría de los niveles estriba en probar que efectivamente la elaboración en profundidad de la informa- ción garantiza su paso a la memoria a largo plazo. Con este objetivo, a partir del artículo de Craik y Lockhart se llevan a cabo una serie de estudios de entre los cuales es quizá el de Craik y Tulving -que también se reproduce a continuación- el más representativo. En relación con este artículo habría que hacer hincapié en tres aspectos cuyo comentario nos va a permitir entrar en algunas de las cuestiones más debatidas en el terreno del aprendizaje humano y la memoria. En primer lugar, no detendremos en el procedimiento del aprendizaje incidental con tareas de orientación como método experimental para investigar la naturaleza de los procesos cognitivos. En segundo lugar, se discutiră el concepto de profundidad de procesamiento para, en tercer lugar, pasar a examinar la noción de memoria episódica en el marco de la teoría de los niveles.

En relación con el primer punto, se trata en principio de que las actividades de codificación pasen a ser controladas por el experimentador para hacer que el sujeto procese la información a un determinado nivel. De esta manera puede establecerse la relación entre el nivel de procesamiento y la ejecución en la memoria o, lo que es lo mismo, el nivel de procesamiento y el grado en que la información pasa a la memoria a largo plazo. Si cuanto más profundo es el nivel de procesamiento mayor resulta ser la retención, entonces la teoría queda confirmada. Para conseguir controlar las actividades de codificación, se les impone. a los sujetos en la fase de adquisición una tarea - la tarea de orientacióncuya realización conlleva procesar el material a un nivel de profundidad preestablecido. En los primeros trabajos se creaba experimentalmente una situación de aprendizaje incidental, es decir, aquella en la que el sujeto desconocía, durante la adquisición, que iba a ser sometido a una prue ba de memoria posterior, aunque, como ya se sabía y confirman Craik y Tulving, el procedimiento incidental o intencional no modifica básicamente los resultados. 
En realidad el procedimiento de aprendizaje incidental con tareas de orientación, que se describe exhaustivamente en el artículo de Craik y Tulving, no es más que una extensión del procedimiento clásico en los estudios de aprendizaje humano que esencialmente descansa en el uso de instrucciones. Es justamente esta la principal característica que distingue, desde el punto de vista experimental, los trabajos con sujetos animales de los trabajos con sujetos humanos. Cuando el psicólogo trabaja con animales tiene que suplir las instrucciones con la manipulación que hace de la situación experimental (Marx, 1977), pero esto introduce un factor fundamental que establece una diferencia primordial entre los estudios con sujetos humanos y los estudios con sujetos animales: el diferente tratamiento que se hace de los aspectos motivacionales. Si un experimentador animal quiere que se cumplan las instrucciones que transmite a través de la manipulación que hace del medio, tiene que plantearse seriamente el problema de motivar a sus sujetos. En el caso de los sujetos humanos se supone que están genéricamente motivados para realizar la tarea que se les encomiende. Por esta razón en la historia de las investigaciones con sujetos humanos no es difícil detectar numerosos casos en los que lo que éstos hacen carece casi por completo de sentido. De ahí la sospecha de muchos teóricos de que probablemente las actividades cognitivas que lleva a cabo una persona en el laboratorio se adecúan a las tareas a las que allí se le obliga y a las condiciones en las que las realiza, pero no tienen nada que ver con lo que generalmente hace en su vida real. En este sentido el procedimiento del aprendizaje incidental con tareas de orientación permite replantearse el problema de la «validez ecológica» de las investigaciones con sujetos humanos, siempre y cuando las tareas que se impongan sirvan para reproducir procesos cognitivos funcionalmente importantes. Este enfoque es el único que puede impedir que este tipo de experimentos, co- mo los que se describen en el artículo de Craik y Tulving, den lugar a teorías que expliquen únicamente fenómenos que se producen en situaciones anómalas y artificiales, como es el caso del aprendizaje de pares asociados o el paradigma de Sternberg (Lockhart, 1979).

Las tareas de orientación, como método experimental en el marco de la teoría de los niveles, han abierto nuevas perspectivas en la investigación acerca de los procesos cognitivos con diversos sujetos y en diversas situaciones. Valiéndose de esta técnica, Cermak y Reale (1978), por ejemplo, han probado que los alcohólicos aquejados del síndrome de Korsakoff deben su amnesia, al menos en parte, a deficiencias en el procesamiento. Algo similar ocurre con los pacientes esquizofrénicos. Cuando a estos pacientes se les compele a procesar en profundidad desaparece su déficit de memoria característico (Kayton y Peterson, 1976). En los estudios con niños, el procedimiento de las tareas de orientación permite comprobar la hipótesis de que las estrategias para recordar van desarrollándose con la edad. En términos generales, los adultos recuerdan igual una lista de palabras cuando se les obliga a realizar una tarea de orientación semántica en una situación de aprendizaje incidental que cuando se les instruye para que aprendan esa misma lista en una situación de aprendizaje intencional. En el caso de los niños esto no ocurre así. Recuerdan mejor en situaciones de aprendizaje incidental con tareas facilitadoras que en situaciones de aprendizaje intencional (Murphy y Brown, 1975). Esto significa que los niños no emplean por sí mismos estrategias adecuadas cuando intentan aprenderse un material, pero si mediante una tarea de orientación se pone en juego la estrategia oportuna, entonces recuerdan igual que los adultos. Hasta los 11 ó 12 años los niños no solamente no emplean espontáneamente estrategias mnemónicas efectivas, sino que desconocen el modo de operar del sistema de memoria. El desarrollo que se produce con 
la edad implica, pues, tanto un desarrollo de la memoria como de la matememoria (Kreutzer, Leonard y F:avell, 1975). A los niños de 4 años, por ejemplo, les parece más fácil aprenderse una lista de palabras si éstas están fonéticamente relacionadas que si están semánticamente relacionadas. Sin embargo, como se ha dicho, cuando se les impone una tarea de orientación, que les obliga a analizar semánticamente las palabras, se comprueba que las recuerdan mejor que si previamente realizan un análisis fonético. Para entender mejor esta paradoja es necesario distinguir entre los procesos de memoria deliberados y los procesos automáticos (Naus y Halasz, 1979). La mayor parte de las cosas que recordamos no han sido almacenadas porque lo hayamos planeado así. Para seguir con nuestro ejemplo, con algunos números de teléfono suele ocurrir que nos damos cuenta de haberlos aprendido sencillamente porque un buen día ya no tenemos necesidad de mirarlos en la agenda, sin que hayamos tenido que realizar ningún esfuerzo para aprenderlos. En otros casos, sin embargo, ponemos en marcha una serie de estrategias para memorizar un teléfono. Lo que sucede con los niños es que el desarrollo de los procesos deliberados de memoria tienen un mayor alcance que el desarrollo de los procesos automáticos que dependen de factores estructurales como la ampliación de la memoria semántica. De esta forma, si se consigue introducir en los procesos automáticos actividades que los adultos llevan a cabo en los procesos deliberados, mediante el uso de tareas de orientación, la ejecución en la memoria mejora notablemente.

Es importante descubrir qué es lo que hacen las personas cuando tratan de memorizar algo, no solamente porque en algunas ocasiones puede suceder que no se lleven a cabo las actividades más adecuadas, sino porque de esta manera sabremos algo más acerca del funcionamiento de la memoria. Una posibilidad es preguntarle a la gente qué es lo que hace, pero los métodos introspectivos se han mostrado ineficaces en la investigación psicológica. El procedimiento del aprendizaje incidental constituye una alternativa. Si conseguimos que una persona recuerde lo mismo un material cuando realiza sobre él una tarea diseñada por nosotros que cuando le decimos simplemente que intente recordarlo, cabe suponer que ha hecho lo mismo o algo muy parecido. Las tareas de orientación, entonces, pueden servir para investigar por qué las estrategias que se ponen en marcha en situaciones intencionales son eficaces. Pero también el uso de tareas de orientación en situaciones incidentales permite estudiar cómo afectan las distintas variables a los procesos de memoria automáticos. De hecho se sabe que las personas emplean una gran variedad de estrategias cuando se proponen memorizar algo, incluso cuando hacen de sujetos en un laboratorio. Pero lo importante no es describir con exactitud estas estrategias. Esta es una labor que han venido realizando todos aquellos que desde los antiguos griegos se han dedicado al arte de la mnemotecnia. Lo importante, como se ha dicho, es saber por qué funcionan esos trucos para mejorar la memoria. En general todas estas técnicas para recordar comparten una característica esencial y es que proporcionan un significado a un conjunto de items que de otra manera carecen de relación entre sí. Por eso, cuando obligamos a un sujeto a realizar un análisis semántico sobre un material verbal, lo recuerda posteriormente mejor que si hubiera realizado un análisis fonético. En el primer caso ha hecho algo parecido a lo que suele hacer en su vida cotidiana cuando emplea uno de sus trucos para recordar.

Los psicólogos habían comprobado ya que codificar un material semántico aumentaba las posibilidades de recuerdo ( $\mathrm{Hy}$ de y Jenkins, 1969), cuando Craik y Lockhart propusieron que ello se debía a que así se procesaba a una mayor profundidad. Ciertamente esta propuesta podría quedar reducida a un mero cambio de ter- 
minología, si no se aclara qué se quiere decir con procesar a un mayor nivel de profundidad que es, por otra parte, el segundo punto que nos proponíamos discutir. Como ya se ha señalado, un nivel de procesamiento más profundo es aquel que hace que la información sea más compatible con las estructuras a largo plazo con que cuenta un individuo, pero habría que describir cuáles son los posibles niveles y habría que aclarar por qué una mayor compatibilidad entre la nueva información que entra y la información almacenada da como resultado que la primera sea más memorizable.

Al comienzo, como puede apreciarse en el artículo de Craik y Lockhart, se asumía que el procesamiento de la información tenía lugar a lo largo de una serie de estadios fijos. Una palabra escrita, por ejem. plo, se analizaría recorriendo toda una jerarquía de niveles que se sucederían serialmente de abajo a arriba desde, pongamos por caso, del análisis puramente gráfico, pasando por el análisis fonético, hasta el análisis semántico. Así, cada input del tipo que fuera podría analizarse de acuerdo con una jerarquía fija de niveles. El efecto de las tareas de orientación sería, por así decirlo, el de parar el procesamiento a un determinado nivel. Al encomendar a un sujeto una tarea que exige un análisis puramente fonético de una lista de palabras se suponía que éste comenzaba por entregarse a un análisis gráfico, para pasar al análisis fonético en el que se detenía sin llevar a cabo, por tanto, ningún tipo de análisis semántico. Se suponía, además, que los niveles de profundidad podían inferirse de los niveles de complejidad lógica. De este modo, lo único que quedaba por hacer era comprobar qué grado de complejidad tenía que alcanzar el análisis lógico para que el nivel fuera cualitativamente más profundo desde el punto de vista psicológico.

Esta forma de entender las cosas resultó ser insostenible por varias razones. En primer lugar, porque no tenía en cuenta al- gunos fenómenos ya conocidos como el efecto Stroop. Desde hacía tiempo se sabía que cuando se pedía a una persona que dijera lo más rápidamente posible de qué color era la tinta con que estaban escritas unas palabras, respondía más despacio o se equivocaba; si la palabra era el nombre de algún color que no coincidía con el de la tinta con que estaba escrita, como cuando la palabra AZUL, por ejemplo, estaba escrita en rojo. Con ello se probaba que, aunque la tarea no requería pasar a un análisis del significado de las palabras, este se procesaba de modo automático y de ahí la interferencia producida. Aparte del efecto Stroop, se habían realizado algunos otros estudios sobre atención selectiva que también evidenciaban el procesamiento automático a niveles profundos, con lo cual quedaba invalidado, en parte, el modelo propuesto. Había, además, otro problema con esta primera formulación de la teoría. Un número de experimentos como los realizados por Savin y Bever (1970) en los que sus sujetos identificaban con mayor rapidez unas sílabas que las letras com. ponentes, mostraban que en algunas ocasiones las unidades lingüísticas de orden superior se procesaban antes que las unidades de orden inferior. Por otra parte, Jonides y Gleitman (1972) demostraron que el símbolo "O» se reconocía más rápidamente en un conjunto de letras cuando se definía como cero que cuando se definía como la letra o. Como la forma física del input era exactamente igual, lá mayor rapidez en la identificación sólo podía deberse a un procesamiento categorial previo o simultáneo al reconocimiento de las características físicas. Estos dos trabajos mencionados pueden servir como muestra de todo un cúmulo de estudios que llevaron a muchos teóricos a la convicción de que junto a un procesamiento de abajo a arri$\mathrm{ba}$, inducido por los datos (data driven) coexiste un procesamiento de arriba a abajo, inducido conceptualmente (conceptually-driven). Había que abandonar, pues, la idea de que el procesamiento tiene lu- 
gar únicamente de abajo a arriba a través de una jerarquía de estadios fijos.

Para superar este punto muerto se intentó buscar una variable observable y cuantificablé que estuviera relacionada con los distintos grados de profundidad a que se analiza un estímulo. El tiempo de procesamiento fue la primera variable que se sugirió en este sentido. La hipótesis era que cuanto más profundo fuera el análisis del input, mayor sería el tiempo que se tardaría en llevarlo a cabo. De esta manera el nivel de profundidad de procesamiento podría determinarse de un modo experimental, al margen de cualquier consideración teórica. Como Craik y Tulving demuestran en uno de sus experimentos, esto no fue posible porque, por efecto de la práctica, algunas de las operaciones que se exigen en el procesamiento en profundidad de un input están automatizadas, con lo cual en algunas ocasiones el procesamiento a mayor profundidad consume menos tiempo. La segunda variable que se propuso fue la de la cantidad de recursos cognitivos consumidos o, de acuerdo con Kahneman (1973), la cantidad de atención o energía cognitiva que se vuelca sobre un input. La capacidad de procesamiento de las personas es limitada y los estudios de atención selectiva habían demostrado que el análisis físico de un estímulo requiere una menor utilización de dicha capacidad que su análisis semántico. Por lo tanto, la cantidad de atención que se dedica a un estímulo está relacionada con el nivel de profundidad a que se le procesa. La capacidad consumida en una operación cognitiva no es, sin embargo, una variable fácil de medir. Una posibilidad, derivada del trabajo de Sokolov (1963), podría ser la de medir las respuestas de orientación ligadas a estados de alerta, pero estas respuestas, ya sean viscerales o corticales, no están únicamente relacionadas con la actividad cognitiva, sino que dependen también de factores emocionales. Johnston, Greenberg, Fisher y Martin (1970) validan experimentalmente un procedimiento alternativo con- sistente en pedir a los sujetos que realicen dos tareas simultáneas, una de las cuales - la tarea subsidiaria - sirve para medir la cantidad de recursos que consume la tarea principal. La tarea subsidiaria, que generalmente consiste en detectar una señal, se ve afectada por la cantidad de atención que exige la tarea principal, de forma que puede medirse dicha cantidad de atención según sea el tiempo de reacción a la señal.

Eysenck y Eysenck (1979), valiéndose de esta técnica, han conseguido demostrar que, aunque algunas tareas no semánticas complejas requieren mayor tiempo de procesamiento que algunas tareas semánticas simples - como ya habían mostrado Craik y Tulving en su experimento 5-, consumen, sin embargo, una menor cantidad de recursos. Por lo tanto, la cantidad de atención consumida por una tarea es un mejor índice de profundidad que el tiempo de procesamiento. A pesar de todo no es un índice totalmente fiable justamente por la misma razón que no lo es el tiempo de procesamiento. El procesamiento automático no sólo afecta al tiempo de procesamiento, sino que afecta también a la atención, puesto que una operación automática consume menos recursos. Como la automatización de una operación se logra con la práctica puede suceder que algunos análisis más profundos requieran una menor atención que algunos análisis superficiales. La activación del significado de las palabras, por ejemplo, a que nos hemos referido anteriormente, es una operación automática desde los siete años aproximadamente (Hasher y Zacks, 1979).

La investigación sobre la relación entre el nivel de profundidad de procesamiento y la atención ha sido muy útil para aclarar algunos extremos acerca de cómo tiene lugar el procesamiento, pero no ha sido decisiva a la hora de especificar el concepto de nivel de profundidad. Como consecuencia de ello se han intentado otros caminos para ampliar y caracterizar con más precisión dicho concepto. Se pensó que una forma de abordar el problema sería 
preguntarse por qué razón el procesamiento a mayor profundidad daba como resultado una mayor retención. Lo primero que se adujo al respecto es que el procesamiento en profundidad y en concreto el análisis semántico de una palabra, por ejemplo, producía una huella más distintiva, puesto que en general el número de rasgos semánticos es mucho mayor que, pongamos por caso, el número de rasgos fonéticos (Wickelgren, 1973). Cuanto mayor sea el número de rasgos en una dimensión de análisis mayor será la probabilidad de que la huella resultante sea muy diferenciada con respecto a otras posibles huellas en esa misma dimensión. Consecuentemente será también menos vulnerable a la interferencia que, como se recordará, es una de las causas por las cuales una huella se pierde de la memoria a largo plazo. Así se explicaría que la información visual se retenga mejor que esa misma información presentada verbalmente (7), puesto que el número de rasgos posibles en una dimensión visual es mayor que en una dimensión verbal (Nelson y Reed, 1976). Ahora bien, si la distintividad de una huella dependiera únicamente del número de atributos de la información no haría falta recurrir a la noción de profundidad, ya que cabe pensar en dimensiones de análisis superficiales con multitud de rasgos. Eysenck (1979), por ejemplo, emprendió una serie de estudios en los que demostró que el procesamiento atípico, y por tanto distintivo, de una palabra a nivel fonético produce una mayor retención. Ocurre, sin embargo, que la distintividad de una huella no depende estrictamente hablando de que la información recibida sea muy diferenciada. Goldstein y Chance (1971) eligieron dos materiales - caras y copos de nieve - que objetivamente son muy distintivos y trataron de averiguar cuál de ellos se memorizaba mejor. Como era de esperar, encontraron que las caras se recordaban mejor, aunque los copos de nieve son muy diferentes entre sị, incluso más que las caras. La razón de este resultado estriba en que hay numerosos atributos que diferencian una cara de otra que podemos relacionar con otras cosas, sin embargo la diferencia entre dos copos de nieve no nos dice nada.

La distintividad de una codificación es esencial para retener la información, pero debe entenderse como un resultado de la elaboración con que se procesa la información. La elaboración a su vez depende del sentido que la información tenga para el sujeto y de su capacidad. Aunque Craik y Tulving también añaden al de profundidad el concepto de elaboración y el de amplitud de procesamiento, no llegan a establecer una relación clara entre ellos. El problema radica en que, aunque para estos autores un análisis en profundidad es un análisis semántico, tienden a identificar la noción de significado psicológico con la noción de significado procedente de la Lingüística. Desde un punto de vista psicológico, las cosas no tienen sentido en sí mismas, sino para el sujeto. El sentido de la información depende no sólo de la información misma, sino también de las estructuras de conocimiento del sujeto y de las metas que se proponga alcanzar. Como han señalado Bransford y McCarrell (1957), el análisis del significado semántico de ls palabras puede no ser lo que más sentido tenga en una determinada situación y en relación con una tarea dada. La frase «coche viene antes de puerta» sólo tiene sentido si se prescinde del significado de las palabras «coche» $y$ «puerta» y se repara únicamente en que la primera precede a la segunda en el diccionario. Esto último, claro está, si la tarea en la cual se inscribe esa frase consiste en buscar palabras en el diccionario.

El significado depende, ciertamente, del material y de la tarea, pero también depende de la experiencia previa del individuo. Las características de los diferentes vinos tienen sentido para un enólogo, pero no para un abstemio. Las estructuras de conocimiento del enólogo adquiridas a lo largo de sus experiencias con los vinos le 
permiten procesar en profundidad el conjunto de rasgos que configuran un determinado vino. La mayor o menor pericia del sujeto impone un límite para procesar la información en profundidad y a su vez determina su capacidad para retenerla. Los jugadores de ajedrez son capaces de recordar las posiciones de las fichas en el tablero con mucha más facilidad que un principiante, pero cuando las fichas están colocadas al azar su capacidad disminuye hasta el nivel de cualquier otro sujeto (Chase y Simon, 1973).

Este enfoque del significado es la aportación fundamental de un grupo de teóricos que se llaman a sí mismos neogibsonianos, porque, como Gibson, piensan que la capacidad de conocimiento es la capacidad de diferenciación y que cuando alguien adquiere el conocimiento lo que en realidad adquiere son destrezas que le permiten diferenciar la información proveniente del medio. Las experiencias concretas sólo son útiles en la medida en que de ellas se derive la adquisición de algún conocimiento que pueda transferirse a situaciones nuevas, pero no por el recuerdo que guardamos de ellas. Para estos autores recordar los acontecimientos no tiene ningún valor; sólo importa la transformación de las estructuras de conocimiento. Es como si las personas dispusieran de una capacidad para recordar episodios que no sirviera para nada. Habría que preguntarse, entonces, cuál es el papel que desempeñan nuestros recuerdos de acontecimientos concretos en la actividad cognitiva. Esta pregunta nos lleva al tercer punto que nos proponíamos examinar más arriba y que hacía mención al concepto de memoria episódica.

En contra de lo que se había venido pensando hasta hace muy poco tiempo, todo lo que se almacena en la memoria a largo plazo no está representado de un modo abstracto como ocurre con el concepto de «mesa» o el de «casas. Hay algunos acontecimientos concretos, algunos episodios que perviven en nuestra memoria a lo largo del tiempo. Tulving (1972), tratando de describir esta diferencia, introdujo la noción de memoria episódica para contraponerla a lo que desde Quillian (1968) se conocía con el nombre de memoria semántica. Lo que Tulving vino a decir es que lo que los sujetos aprendían en un laboratorio, cuando se les presentaba una lista de palabras, no eran las palabras en sí, sino el hecho de que esas palabras aparecieran en la lista. En estos experimentos no se modificaban las estructuras de conocimiento - la memoria semán. tica- de los sujetos, puesto que las pala. bras de la lista eran previamente conocidas. Esta es una de las razones por las que las investigaciones que se llevaban a cabo en los laboratorios de psicología sobre el aprendizaje humano no tenían ninguna incidencia en la Educación. Este tipo de investigaciones, pues, eran estudios de memoria episódica; de lo que ya antes Bertrand Russell había llamado «pura memoria». Si se nos permite ir más atrás todavía en los antecedentes de esta diferencia entre la memoria episódica y la memoria semántica, podría afirmarse que se corresponde con la distinción kantiana entre los juicios analíticos y los juicios sintéticos. Desde el punto de vista psicológico la noción de memoria episódica permite aclarar algunas cuestiones en torno a lo que los organismos aprenden en el curso de sus experiencias. En el campo de la Psicología animal, por ejemplo, los estudios sobre la memoria episódica han dado lugar a reinterpretaciones globales de muchos de los fenómenos que tradicionalmente se habían estudiado en el marco del condicionamiento pauloviano (8).

Una vez hecha esta distinción, una de las tareas que los investigadores tenían que llevar a cabo consistía en averiguar cuáles son los factores qu determinan la recuperación de la información episódica. Tulving y colaboradores (Tulving y Thomson, 1973; Tulving y Watkins, 1975; Tulving y Wiseman, 1975) al probar su «Principio de especificidad de codificación», demostraron 
que el factor decisivo es la semejanza entre el contexto en la codificación y el contexto en la recuperación. Es como cuando no reconocemos a una persona que hemos visto muchas veces, al encontrárnosla en una situación totalmente diferente. En el caso de nuestro ejemplo de alguien que se aprende un número de teléfono, puede que, aunque se lo sepa, no sea capaz de reconocerlo en una matrícula de coche. De todas formas es imposible encontrar un ejemplo perfecto de memoria episódica, sencillamente porque la «Pura memoria» no existe. Tulving concibió inicialmente dos almacenes independientes en la memoria a largo plazo, uno de memoria semántica y otro de memoria episódica, pero esto no se corresponde con los datos de que disponemos. De hecho, la memoria semántica y la memoria episódica están intimamente relacionadas. Si le preguntamos a una persona que qué hizo al levantarse por la mañana, aparentemente su respuesta dependerá de su capacidad para recuperar la huella episódica de lo que ocurrió esa mañana. Sin embargo, esto no es del todo exacto. A lo mejor no tiene una huella clara de que se lavó los dientes, así y todo afirmará que lo hizo porque si no lo hubiera hecho se acordaría. Lo que a simple vista sólo puede contes. tarse en base a los datos recolectados en la memoria episódica, se responde en la práctica recurriendo también a la memoria semántica. Las primeras cifras de los teléfonos suelen coincidir en cada población o, al menos, pertenecen a una gama reducida de posibilidades. En algunos sitios, por ejemplo, un número de teléfono no puede empezar por 8 , de forma que cuando recuperemos las primeras cifras de un telé. fono estamos empleando nuestra memoria semántica. Por lo tanto, los datos episódi$\cos$ no se recuperan al margen de la memoria semántica ni consecuentemente el sistema episódico es independiente del sistema semántico.

La relación entre estos dos sistemas puede concebirse de un modo más general. El aprendizaje podría entenderse como un proceso mediante el cual la información fuera desprendiéndose de sus aspectos contextuales. Cuanto más contextualizada esté la información, más estará situada en el extremo episódico y cuanto más descontextualizada más estará en el extremo semántico. Lo que se localiza en el extremo espisódico serían los recuerdos mientras que en el otro extremo se localizaría lo que podríamos llamar «aprendidow. Craik y Jacoby (1979) proponen una descripción según la cual el continuo episódico-semántico puede ejemplificarse de acuerdo con un modelo jerarquizado en el cual los nodos inferiores están formados por acontecimientos muy ligados a un contexto. En los nodos a un nivel superior se representa la información común a varios nodos inferiores y para ello parcialmente descontextualizada. Conforme se avanza en la jerarquía los nodos contienen una información más abstracta, aunque su origen está en las experiencias concretas por las que ha atravesado el individuo y que inicialmente se representan como episodios. Cuanto más abstracta es la información representada más utilizable es para interpretar la nueva información.

Los conocimientos que poseemos nos sirven de prototipos a la hora de interpretar la nueva información (Norman y Bobrow, 1979). Pero estos prototipos nunca se corresponden exactamente con los datos, generándose así una descripción que, junto con los procedimientos empleados para llegar a ella, es lo que constituye la huella de memoria episódica, independientemente de que las estructuras semánticas se hayan transformado para acomodarse a la información procedente del medio. Si el prototipo elegido es abstracto estará relacionado con otras unidades de memoria y, por tanto, será muy fácil elaborar la información. Si por el contrario es muy dependiente del contexto sólo podrá relacionarse con la información que entra de un modo limitado. 


\section{Notas}

(1) Ver la entrevista con Norman publicada en el número 1 de esta misma revista.

(2) En nuestros días, la tendencia se ha invertido y los investigadores del aprendizaje animal están incorporando la estructura conceptual procedente del aprendizaje humano y la memoria. Las nuevas corrientes cognitivas han irrumpido de un modo decidido en el campo de la Psicología animal, reinterpretando de nuevo los trabajos que desde el final de la Primera Guerra mundial hasta mediados de los años 50 habían constituido el grueso de la ciencia psicológica. Para un tratamiento más exhaustivo del tema véase Honig (1978).

(3) El almacén sensorial es periférico. Por eso puede prescindirse de él a la hora de describir las actividades en las que interviene el procesador central. Su función es la de prolongar un mensaje sensorial con el fin de dar tiempo al sistema para interpretarlo. Ultimamente se ha cuestionado también la utilidad de considerar la memoria sensorial como un almacén. Para Di Lollo (1977), por ejemplo, la memoria icónica es todo un conjunto de procesos activos de extracción de rasgos.

(4) Ciertamente no es este el punto de vista tradicional acerca de la naturaleza del aprendizaje. Durante varias déçadas un gran número de teóricos, particularmente en el campo del aprendizaje animal, se resistieron a admitir que aprender algo fuera equivalente a adquirir el conocimiento de algo. Más bien pensaban que los organismos se limitaban a aprender respuestas. Actualmente quedan ya pocos psicólogos que sigan definiendo el aprendizaje como un cambio en la conducta. Es más, como ha demostrado Rescorla (1978), la conducta de un animal ni siquiera es en todos los casos el mejor índice de lo que éste aprende en su interrelación con el medio.

(5) En el artículo de Atkinson y Shiffrin (1968) se discuten ampliamente los procesos de control, pero sorprendentemente estos procesos no se incorporan al modelo que proponen. Se concentran únicamente en algunas propiedades del sistema de modo que sea posible hacer algunas predicciones, sin que en sus análisis de los datos se reflejen cuestiones tan importantes como las estrategias de codificación del sujeto (Newell, 1972).

(6) Una crítica más actualizada de las teorías multialmacén se encuentra en Craik y Levy (1976).

(7) La idea de que la información visual se retiene mejor que la información verbal tiene mucho que ver con el célebre proverbio chino de que una imagen vale por mil palabras. Su demostración experimental corrió a cargo de Shepard (1967), Paivio, Rogers y Smythe (1968) y Standing, Conezio y Haber (1970).

(8) Para Wagner (1976) o Whitlow (1976) el EC puede entenderse como una clave de recuperación de la representación del EI. Como, según el principio de especificidad de codificación, el factor determinante en la recuperación de una huella episódica es la semejanza entre el contexto en la codificación y el contexto en la recuperación, las claves de recuperación serán efectivas en la medida en que reproduzcan el contexto de la adquisición en la recuperación. En el caso del condicionamiento clásico los emparejamientos EC-EI convierten al EC en una clave de recuperación que será tanto más efectiva cuanto mayor sea el número de emparejamientos.

\section{Referencias}

ATKINSON, R, C., y CROTHERS, E. J. A comparison of paired-associate learning models having different acquisition and retention axioms. Journal of Matbematical Psychology, 1964, $1,285-315$.

ATKINSON, R. C., y SHIFFRIN, R. M. Human memory: A proposed system and its control processes. En K. W. Spence y J. T. Spence (eds.). The psychology of learning and motivation (Vol. 2). Nueva York: Academic Press, 1968.

BJORK, R. A. Short-term storage: The ordered output of a central processor. En F. Restle, R. M. Shiffrin, N. J. Castellan, H. R. Lindman y D. B. Pisoni (eds.) Cognitive theory. (Vol. 1). Hillsdale, N. Jersey: Lawrence Erlbaum Associates, 1975.

BOWER, G. H. An association model for response and training variables in paired-associate learning. Psychological Review, 1962, 69, 34-53.

BRANSFORD, J. D., y McCARRELL, N. W. A sketch of a cognitive approach to comprehension: Some thoughts about understanding what it means to comprehend. En W. Weimer y D. Pa- 


\section{Estudios}

lermo (eds.), Cognition and the symbolic processes. Hillsdale, N. Jersey: Lawrence Erlbaum Associates, 1975.

BROADBENT, D. E. Perception and communication. Nueva York: Pergamon Press, 1958.

BROWN, J. A. Some tests of the decay theory of inmediate memory, Quarterly Journal of Experimental Psychology, 1958, 10, 12-21.

CERMAK, L. S., y REALE, L. Depth of processing and retention of words by alcoholic Korsakoff patients. Journal of Experimental Psychology: Human Learning and Memory, 1978, 4, 165-174:

CHASE, W. G., y SIMON, H. A. The mind's eye in chess. En W. G. Chase (ed.), Visual Information processing. Nueva York: Academic Press, 1973.

CRAIK, F. I. M., y JACOBY, L. L. A process view of short-term retention. En F. Restle, R. M. Shiffrin, N. J. Castellan, M. R. Lindman y D. B. Pisoni (eds.) Cognitive theory (Vol. 1). Hillsdale, N. Jersey: Lawrence Erlbaum Associates, 1975.

CRAIK, F. I. M., y JACOBY, L. L. Elaboration and Distinctiveness in Episodic Memory. En L. G. Nilsson (ed.) Perspectives on memory research. Hillsdale, N. Jersey: Lawrence Erlbaum Associates, 1979.

CRAIK, F. I. M., y LEVY, B. A. The Concept of Primary Memory. En W. K. Estes (ed.) Handbook of Learning and Cognitive Processes 8 Vol. 4). Hillsdale, Nueva Jersey: Lawrence Erlbaum ¿ Associates, 1976.

DI LOLLO, V. Temporal characteristics of iconic memory. Nature, 1977, 267, 241-243.

ESTES, W. K. Learning theory and the new kmental chemistry*. Psychological Review, 1960 , 67, 207-223.

EYSENCK, M. W. Depth, Elaboration and Distinctiveness. En L. S. Cermak, y F. I. M. Craik (eds.), Levels of processing in human memory. Hillsdale, Nueva Jersey: Lawrence Erlbaum Associates, 1979.

EYSENCK, M. W., y EYSENCK, M. C. Processing Depth, Elaboration of Encoding, Memory Stores, and Expended Processing Capacity. Journal of Experimental Psychology: Human Learning and Memory, 1979, 5, 472-484.

GOLDSTEIN, A. G., y CHANCE, J. E. Visual recognition memory for complex configurations. Perception \& Psychophysics, 1971, 9, 237-241.

GREENO, J. G. How associations are memorized. En D. A. Norman (ed.), Models of buman memory, Nueva York: Academic Press, 1970.

HASHER, L., y ZACKS; R. T. Automatic and effortful processes in memory. Journal of Experimental Psychology: General, 1979, 3, 356-388.

HONIG, W. K. On the conceptual nature of cognitive terms: and initial essay. En S. H. Hulse, $\mathrm{H}$. Fowler y W. K. Honig (eds.), Cognitive processes in animal behavior. Hillsdale, Nueva Jersey: Lawrence Erlbaum Associates, 1978.

HYDE, T. S., y JENKINS, J. J. The differential effects of incidental tasks on the organization of recall of a list of highly associates words. Journal of Experimental Psychology, 1969, 12, $471-480$.

JENKINS; J. J. Can we have a theory of meaningful memory?En R. L. Solso (ed.) Theories in cognitive Psychology: The Loyola symposium. Potomac, Maryland: lawrence Erlbaum Associates, 1974 .

JOHNSTON, W. A.; GREENBERG, S. N.; FISHER, R. P., y MARTIN, D. W. Divided attention: a vehicle for monitoring memory processes. Journal of Experimental Psychology, 1970, 83, 164-171.

JONIDES, J., y GLEITMAN, H. A conceptual category effect in visual search. Perception \& Psychophysics, 1972, 12, 457-460.

KAHNEMAN, D. Attention and effort. Englewood Cliffs, Nueva Jersey: Prentice Hall, 1973.

KREUTZER, M. A.; LEONARD, C., y FLAVELL, J. H. An interview study of children's knowledge about memory. Monographs of the Society for Research in Child Development, 1975, 40, número 159.

LOCKHART, R. S. Remembering events: discussion of papers by Jacoby and Craik, Battig, and Nelson. En L. S. Cermak y F. I. M. Craik (eds.), Levels of processing in human memory. Hillsdale, Nueva Jersey: Lawrence, Erlbaum Associates, 1979.

MARX, M. H. Research procedures. En M. H. Marx y M. E. Bunch (eds.), Fundamentals and aplications of learning. Nueva York: MacMillan, 1977.

MILLER; G. A. The magical number seven, plus or minus two: some limits on our capacity for processing information. Psychological Review, 1956, 63, $8^{\circ} \cdot 97$. 
MURDOCK, B. B., Jr. The serial effect of free recall. journal of experimental Psychology, $1962,64,482-488$.

MURPHY, M. D., y BROWN, A. L. Incidental learning in preschool children as a function of level of cognitive ana..sis. Journal of Experimental Child Psycholgy. 1975, 19, 509-523.

NAUS, M. J., y HALASZ, F. G. Developmental perspectives on cognitive processing and semantic memory structure. En L. S. Cermak y F. I. M. Craik (eds.), Levels of processing in human memory. Hillsdale, Nueva Jersey: Lawrence Erlbaum Associates, 1979.

NEWELL, A. A. Theoretical exploration of mechanisms for coding the stimulus. En A.W. Melton y E. Martin, Coding processes in buman memory. Washington: Winston, 1972.

NORMAN, D. A., y BOBROW, D. G. Descriptions: an intermediate stage in memory retrieval. Cognitive Psychology, 1979, 11, 107-123.

PAIVIO, A.; ROGERS, T. B., y SMYTHE, P. C. Why are pictures easier to recall than words? Psychonomic Science, 11, 137-138.

PETERSON, L. R., y PETERSON, M. Short-term retention if individual items. Journal of Experimental Psychology, 1959, 58, 193-198.

QUiLliaN, M. R. Semantic memory. En M. Minsky (ed.), Semantic information processing. Cambridge, Massachusetts: MIT Press, 1968.

SAVIN, H. B., y BEVER; T. G. The nonperceptual reality of the phoneme. Journal of Verbal Learning and Verbal Behavior, 9, 295-302.

SHIFFRIN, R. M. Short-term store: tha basis for a memory system. En F. Restle, R. M. Shiffrin,

J N. J. Castellan, H. R. Lindman y D. B. Pisoni (eds.), Cognitive theory. Hillsdale, Nueva Jersey: Lawrence Erlbaum Associates, 1975.

SHEPARD; R. N. Recognition memory for words, sentences and pictures, Journal of Verbal Learning and Verbal Behavior, 1967, 6, 156-163.

SOKOLOV, E. N. Perception and the conditioned reflex. Nueva York: MacMillan, 1963.

STANDING, L.; CONEZIO, J., y HABER, R. N. Perception and memory for pictures: singletrial learning of 2560 visual stimuli. Psychonomic Science, 1970, 19, 73.74.

TULVING, E. Episodic and semantic memory. En E. Tulving y W. Donaldson (eds.), Organization of memory. Nueva York: Academic Press, 1972.

TULVING, E., y THOMSON, D. M. Encoding specificity and retrieval processes in episodic memory. Psychological Review, 1973, 80, 352-373.

TULVING, E., y WATKINS; M. J. Structure of memory traces. Psychological Review, 1975, $82,261-275$.

TULVING, E., y WISEMAN, S. Relations between recognition and recognition failure of recallable words. Bulletin of the Psychonomic Society, 1975, 6, 79-82.

UNDERWOOD, B. J., y KEPPEL, G. One trial learning?Journal of Verbal Learning and Verbal Behavior, 1962, 1, 1-13.

WAGNER, A. R. Priming in STM: An information-processing mechanism for self-generated or retrieval-generated depression in performance. En T. J. Tighe y R. N. Leaton (eds.) Habituation: Perspectives from child development, animal behavior, and neurophysiology. Hillsdale, Nueva Jersey: Lawrence Erlbaum Associates, 1976.

WAUGH, N. C., y NORMAN, D. A. Primary memory. Psychological Review, 1965, 72, 89-104.

WHITLOW, J. W. Jr. The dynamics of episodic processing in pavlovian conditioning. En D. L. Medin, W. A. Roberts y R. T. Davis, Processes of animal memory. Hillsdale. Nueva Jersey: Lawrence Erlbaum Associates, 1977. 